Artigo Original

\title{
Relacionamento de diferentes domínios da atividade física habitual com indicadores de risco cardiovascular em adultos jovens do sexo masculino
}

\author{
Ismael Forte Freitas Júnior ${ }^{3}$ \\ Eduardo Quieroti Rodrigues ${ }^{3}$ \\ Sergio de Sousa ${ }^{3}$ \\ Eduardo Zapaterra Campos ${ }^{3}$ \\ Rômulo Araújo Fernandes ${ }^{1}$ \\ Juliano Casonatto ${ }^{2}$ \\ Pedro Balikian Júnior ${ }^{3}$ \\ ${ }^{1}$ Instituto de Biociências. UNESP - Univ Estadual Paulista- Campus de Rio Claro, \\ Departamento de Educação Física, Rio Claro, SP, Brasil \\ ${ }^{2}$ Centro de Educação Física e Esporte da UEL ,Londrina, PR, Brasil \\ ${ }^{3}$ Faculdade de Ciências e Tecnologia. UNESP - Univ Estadual Paulista- Campus de \\ Presidente Prudente, Departamento de Educação Física, Presidente Prudente, SP, Brasil

\begin{abstract}
Resumo: Objetivos: Avaliar a prática de atividade física por jovens saudáveis em diferentes segmentos (trabalho, lazer e esportes) e relacionar com indicadores de risco para doenças cardiovasculares. Métodos: A amostra foi composta por 32 estudantes de Educação Física, com idade media de 22,6 anos. A adiposidade corporal foi analisada através de circunferência da cintura (CC), índice de massa corporal (IMC) e impedância bioelétrica. Adicionalmente, valores de glicemia em jejum (10-12 horas), pressão arterial sistólica (PAS) e diastólica (PAD) foram coletados. O nível de envolvimento com atividades Físicas foi avaliado por meio de questionário desenvolvido por Baecke et al. (1982). Resultados: As atividades esportivas e de tempo livre não se relacionaram estatisticamente com os indicadores de risco cardiovascular. Porém, as atividades realizadas no trabalho (\%GC, PAS e PAD) a somatória de todos os domínios investigados (PAD e Glicemia) relacionaram-se negativamente com os mesmos. Conclusão: Diferentes domínios da atividade física devem ser considerados quando se analisa o efeito da mesma sobre indicadores de saúde.
\end{abstract}

Palavras-chave: Atividade física. Síndrome metabólica. Adulto. Brasil.

\section{Relationship of different physical activity domains with cardiovascular risk factors among male adults}

\begin{abstract}
Objectives: To analyze different physical activities domains in healthy adults and correlate with cardiovascular risk factors. Methods: The sample was composed by 32 Physical Education undergraduate students, with mean age of 22.6 years old. Adiposity was assessed by waist circumference (WC), body mass index (BMI) and bioelectrical impedance. Additionally, fast glucose (10-12 hours), systolic (SBP) and diastolic (DBP) blood pressure were assessed. Physical activity level was assessed by questionnaire (Baecke et al., 1982). Results: sports activities and leisure time were not related with cardiovascular risk factors. However, job activities were negatively related with \%BF, SBP and DBP. Conclusion: Different physical activity domains should be considered when analyzing its associations with health indicators.
\end{abstract}

Key Words: Physical activity. Metabolic syndrome. Adult. Brazil.

\section{Introdução}

A união de diferentes indicadores de risco para a saúde (obesidade central, dislipidemia, resistência à insulina e hipertensão arterial) constitui a síndrome metabólica (JAMA, 2001). Rotinas sistematizadas de exercícios físicos ou, simplesmente o aumento no gasto energético diário mesmo que não envolvendo exercícios fiscos, são largamente empregados para o controle destes diferentes fatores de riscos à saúde (GUTIN et al., 2005; $\underline{\text { CHRISTOFARO }}$ et al., 2008). Esta larga utilização do exercício físico como tratamento não-farmacológico se dá ao fato de que reduções de gordura corporal total e abdominal, induzidas pelo exercício estão associadas com melhoras significativas na sensibilidade à insulina, pressão arterial e perfil

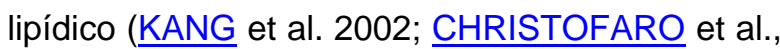
2008). 
Em diferentes grupos etários, a maior prática habitual de atividades físicas constitui um importante fator de proteção ao desenvolvimento de inúmeras patologias, caso dos diferentes componentes da síndrome metabólica citados acima (ORAKZAI et al., 2006; GUTIN et al., 2005; EKELUND et al., 2006; CHRISTOFARO et al., 2008).

A prática de atividades físicas constitui uma variável complexa de ser analisada, pois possui particularidades que precisam ser consideradas quando verificados seus efeitos sobre indicadores de saúde. Linhas mais recentes de pesquisa indicam que os diferentes domínios da prática de atividades físicas (aquelas atividades realizadas no trabalho; em casa; durante horários de lazer; etc.) estão associados de diferentes maneiras com variados indicadores de saúde física e mental (ABU-OMAR et al., 2008). Tais dados salientam que se a atividade física for considerada de maneira total, ou seja, apenas como um somatório de todos os seus domínios, informações importantes podem se perder.

$\mathrm{Na}$ literatura nacional, embora se reconheça o papel relevante da atividade física enquanto agente na prevenção/tratamento de inúmeras patologias, ainda é escassa a realização de estudos analisando o efeito da maior prática habitual de atividades físicas sobre indicadores de saúde (HALLAL et al., 2007). Esta ausência de estudos é mais evidente se contabilizados apenas aqueles que consideraram os diferentes domínios da prática de atividades físicas.

Assim, o objetivo do presente estudo foi analisar a relação de diferentes domínios da prática de atividades físicas com indicadores de risco cardiovascular entre adultos jovens.

\section{Métodos}

\section{Amostra}

Estudo descritivo e analítico com delineamento transversal conduzido durante 0 último trimestre do ano de 2008. A amostra estudada foi composta por 32 estudantes universitários do sexo masculino, todos regularmente matriculados no curso de Educação Física de uma instituição pública de ensino superior. Os critérios para a inclusão dos universitários na amostra do estudo foram baseados em três informações: (i) Ser do sexo masculino e estar devidamente matriculado na presente Universidade; (ii) Não apresentar nenhuma necessidade especial / doença cardíaca que interferira no teste; (iii) Assinar o Termo de Consentimento Livre e Esclarecido.

O estudo seguiu as diretrizes e normas que regulamentam a pesquisa com seres humanos (lei 196/96) e foi aprovado pelo Comitê de Ética em Pesquisa Envolvendo Seres Humanos que está vinculado a universidade na qual foi realizada a pesquisa (Processo 280/2008).

\section{Coleta dos Dados}

Os dados apresentados neste estudo foram coletados no Centro de Estudos e Laboratório de Avaliação e Prescrição de Atividades Motoras (CELAPAM) vinculados ao Departamento de Educação Física da instituição de nível superior responsável pela pesquisa. Em dias previamente agendados, todos os sujeitos compareceram ao laboratório, onde, em temperatura controlada, foram efetuadas as avaliações referentes à glicemia em jejum, impedância bioelétrica, antropometria e pressão arterial, bem como, também foi preenchido o questionário para a prática de atividades físicas.

\section{Domínios da Prática de Atividades Físicas}

O envolvimento com a prática de atividades esportivas foi avaliado por meio do questionário para a prática de atividades físicas desenvolvido por Baecke et al. (1982). Tal instrumento é dividido em seções e, por meio de perguntas fechadas (total de 16 questões), avalia a atividade física habitual em diferentes domínios (Trabalho; Lazer; Esportes; Total [somatória dos três domínios]). Durante a avaliação da prática de atividades físicas, para cada opção de resposta é atribuído um valor fixo e de escala adimensional (presente nos apêndices do instrumento) pelo qual, após o somatório de todos os valores, é possível calcular um escore final para cada um dos domínios da atividade física analisados.

\section{Antropometria}

A massa corporal foi mensurada com a utilização de uma balança digital da marca 
Filizola, com precisão de $0,1 \mathrm{~kg}$ e capacidade máxima de $180 \mathrm{~kg}$. A estatura foi aferida com a utilização de um estadiômetro fixo de madeira com precisão de $0,1 \mathrm{~cm}$ e extensão máxima de dois metros. $O$ índice de massa corporal (IMC) foi calculado por meio da divisão da massa corporal pelo valor da estatura ao quadrado e foi expresso como $\mathrm{kg} / \mathrm{m}^{2}$. O valor da circunferência de cintura (CC) foi adotado como indicador de excesso de tecido adiposo abdominal, sendo as medidas tomadas em duplicata na mínima circunferência entre a crista ilíaca e a última costela, com a utilização de uma fita metálica antropométrica com precisão em milímetros $(\mathrm{mm})$. Todas as medidas antropométricas foram efetuadas seguindo recomendações da literatura (LOHMAN et al., 1988).

\section{Impedância Bioelétrica}

A resistência e a reatância corporal (ohm) foram obtidas com a utilização de um analisador portátil (BIA Analyzer -101Q, RJL Systems, Detroit, EUA). Em cada dia de avaliação, o aparelho foi calibrado antes das avaliações com o uso de um resistor de $500 \mathrm{ohm}$, providenciado pelo próprio fabricante. A BIA foi realizada somente após o esvaziamento da bexiga. Os procedimentos foram realizados com o indivíduo deitado em uma superfície plana de material não condutor de eletricidade (maca) e após a retirada de calçados, meias e qualquer tipo de metal unido ao corpo (brincos, pulseiras, colares, etc.). Os eletrodos transmissores foram colocados no dorso da mão direita, na falange distal do terceiro metacarpo e na superfície anterior do pé direito, na falange distal do segundo metatarso, e ao menos de $5 \mathrm{~cm}$ de distância dos eletrodos receptores, os quais foram colocados entre 0 processo estilóide do rádio e da ulna e entre os maléolos medial e lateral do tornozelo (HEYWARD e STOLARKCZY, 2000).

O percentual de gordura corporal (\%GC-BIA) foi calculado pelo uso de uma equação específica

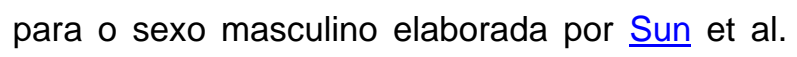
(2003).

\section{Pressão Arterial}

Para a aferição da pressão arterial foi utilizado um aparelho oscilométrico da marca Omron modelo HEM-742, previamente validado (CHRISTOFARO et al., 2009). A pressão arterial foi aferida no braço direito com os indivíduos sentados com pelo menos $5 \mathrm{~min}$ de descanso. Padronizou-se dois minutos de intervalo entre as cada uma das três medidas da pressão arterial e os valores de pressão arterial sistólica (PAS) e diastólica (PAD) foram estimados pela média das três avaliações.

\section{Glicemia em Jejum}

A coleta de sangue foi realizada na própria unidade escolar pelo pesquisador responsável pelo estudo, sendo que, para a realização das análises foi respeitado um jejum de 10-12 horas. Para a dosagem de glicose foi utilizado um aparelho portátil da marca Johnson \& Johnson modelo One Touch Ultra 2, com lancetas One Touch UltraSoft descartáveis e tiras reagentes One Touch Ultra. Anteriormente a coleta das amostras sanguíneas, houve assepsia da superfície da pele com a utilização de um algodão banhado em álcool.

\section{Escore de Risco Cardiovascular}

Os escores de cada um dos indicadores de risco cardiovascular analisados (IMC, CC, \%GCBIA, PAS, PAD e Glicemia) foram ordenados do maior para o menor valor e subdivididos em três grupos: $\mathrm{G} 1$ (33\% com os mais altos valores $[\mathrm{n}=$ 10]), G2 (33\% com valores intermediários os mais baixos valores $[n=11]$ ) e G3 (33\% com os mais baixos valores $[n=11])$. Posteriormente, foi computado o número de vezes que cada indivíduo esteve situado no $\mathrm{G} 1$ de todos os seis fatores de risco cardiovascular e construiu-se um "escore de risco" que variou de 0 (mais baixo risco) a 6 (mais alto risco).

\section{Análise Estatística}

Para as variáveis numéricas, a normalidade do conjunto de dados analisado foi confirmada pelo teste de Komolgorov-Smirnov (K-S). Assim, a estatística descritiva foi composta por valores de média (tendência central) e desvio-padrão (dispersão). A correlação momento produto de Pearson indicou a relação linear entre as variáveis analisadas. Valores de $P$ inferiores a $5 \%$ foram considerados estatisticamente significativos e todas as análises foram realizadas no software estatístico: SPSS 10.0. 


\section{Resultados}

A Tabela 1 apresenta as características gerais da amostra, bem como o relacionamento da idade cronológica com todas as variáveis envolvidas no estudo. A variável idade não se relacionou de maneira significativa do ponto de vista estatístico com todos os indicadores de risco cardiovascular e domínios da atividade física.

Tabela 1. Características gerais da amostra analisada.

\begin{tabular}{|c|c|c|c|c|}
\hline & \multirow{2}{*}{$\begin{array}{c}\text { Correlação c/ Idade } \\
r\end{array}$} & \multirow{2}{*}{ Média (DP) } & \multicolumn{2}{|c|}{ IC95\% } \\
\hline & & & Menor & Maior \\
\hline Idade (anos) & --- & $22,4(3,7)$ & 21,1 & 23,8 \\
\hline IMC $\left(\mathrm{kg} / \mathrm{m}^{2}\right)$ & $-0,06$ & $23,9(3,3)$ & 22,7 & 25,1 \\
\hline $\mathrm{CC}(\mathrm{cm})$ & $-0,11$ & $79,6(7,5)$ & 76,8 & 82,3 \\
\hline$\% G C$ & $-0,11$ & $19,2(5,4)$ & 17,2 & 21,2 \\
\hline PAS (mmHg) & 0,12 & $127(10,4)$ & 123,2 & 130,9 \\
\hline PAD (mmHg) & 0,11 & $74,6(7,8)$ & 71,7 & 77,5 \\
\hline Glicemia (mg/dl) & $-0,16$ & $90,4(12,8)$ & 85,7 & 95,1 \\
\hline \multicolumn{5}{|l|}{ Domínios } \\
\hline Trabalho & $-0,27$ & $2,77(0,7)$ & 2,49 & 3,04 \\
\hline Esporte & $-0,06$ & $4,33(1,6)$ & 3,72 & 4,95 \\
\hline Tempo Livre & $-0,06$ & $2,71(0,8)$ & 2,41 & 3,01 \\
\hline Total & $-0,13$ & $9,81(2,5)$ & 8,88 & 10,7 \\
\hline
\end{tabular}

$\mathrm{PAS}=$ pressão arterial sistólica; $\mathrm{PAD}=$ pressão arterial diastólica; $\mathrm{CC}=$ circunferência de cintura.

$\mathrm{Na}$ Tabela 2 são apresentados os valores de correlação entre os indicadores de risco cardiovascular e os diferentes domínios da atividade física. As atividades realizadas no trabalho apresentaram relação negativa e significativa com o \%GC indicado pela impedância bioelétrica, bem como com ambos indicadores hemodinâmicos utilizados no estudo (PAS e PAD).

Tabela 2. Relacionamento entre domínios da atividade física e indicadores de risco cardiovascular.

\begin{tabular}{lcccc} 
& Trabalho & \multicolumn{2}{c}{ Domínios da Atividade Física } & Total \\
& $r$ & Esporte & Tempo livre & r \\
\hline IMC $\left(\mathrm{kg} / \mathrm{m}^{2}\right)$ & $-0,24$ & $-0,06$ & $-0,03$ & $-0,12$ \\
CC $(\mathrm{cm})$ & $-0,26$ & $-0,12$ & $-0,14$ & $-0,19$ \\
\%GC & $-0,35^{*}$ & $-0,21$ & $-0,15$ & $-0,28$ \\
PAS (mmHg) & $-0,35^{*}$ & $-0,26$ & $-0,18$ & $-0,32$ \\
PAD (mmHg) & $-0,41^{*}$ & $-0,31$ & $-0,16$ & $-0,36^{*}$ \\
Glicemia (mg/dl) & $-0,25$ & $-0,30$ & $-0,28$ & $-0,36^{*}$ \\
\hline
\end{tabular}

${ }^{*}=\mathrm{p}<5 \% ; \mathrm{IMC}=$ índice de massa corporal; PAS= pressão arterial sistólica; $\mathrm{PAD}=$ pressão arterial diastólica; $\mathrm{CC}=$ circunferência de cintura.

As atividades realizadas no Esporte e durante horários de Tempo Livre não se relacionaram com nenhum dos indicadores de risco cardiovascular analisados. Porém, a atividade física Total, que é a somatória de todos os domínios investigados, relacionou-se de maneira negativa com a glicemia em jejum e PAD.

Com base nos valores mais elevados em cada um dos indicadores de risco, criou-se um escore de risco cardiovascular e analisou-se a relação do mesmo com os diferentes domínios da prática de atividades físicas (Figura 1)

O único domínio da prática de atividade física que se relacionou estatisticamente com o escore de risco criado foi o do Trabalho. 

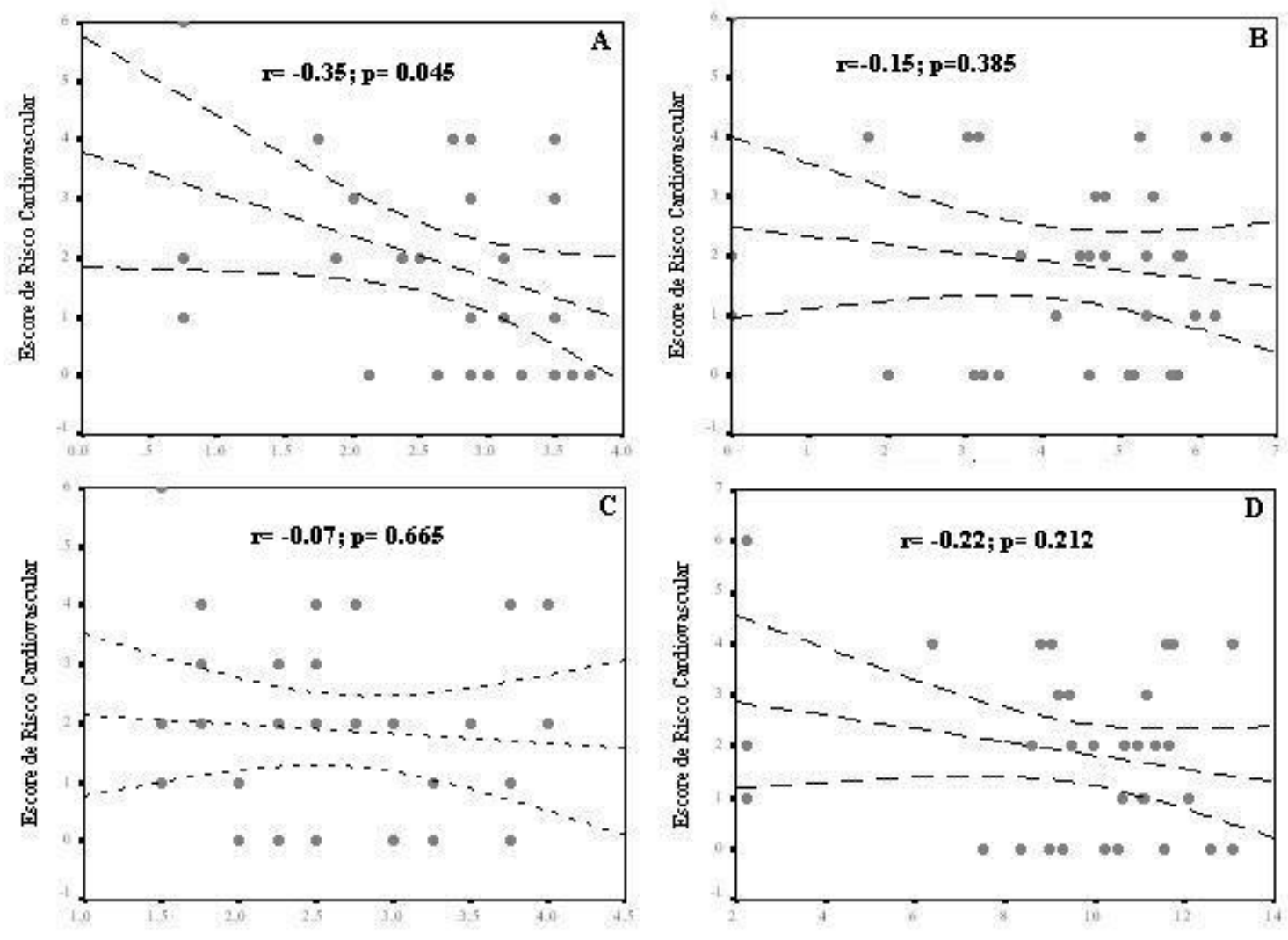

Figura 1. Relacionamento entre diferentes domínios da prática de atividade física (A) Trabalho, (B) Esporte, (C) Tempo Livre e (D) Total com indicadores de risco cardiovascular.

\section{Discussão}

Estudo de delineamento transversal que envolveu adultos jovens do sexo masculino, no qual se analisou a relação de diferentes domínios da prática de atividades físicas com indicadores de risco cardiovascular. Foi detectado que o relacionamento da atividade física com tais indicadores de risco a saúde varia de acordo com o domínio investigado.

A maior aptidão física de um indivíduo é associada com a prática de exercícios físicos regulares (GUTIN et al. 2005) e esta relação, pode ser considerada como um dos principais fatores que tornam a atividade física um importante indicador de promoção à saúde física. Os resultados estão em linha com tal afirmação e com observações anteriores que indicam relações negativas entre estes indicadores de risco cardiovascular e a maior prática de atividades físicas (GUTIN et al., 2005; EKELUND et al., 2006). Tais resultados corroboram com a idéia de que a maior prática de atividades físicas pode constituir um fator determinante para redução dos riscos de doenças de origem crônico-degenerativa.
A relação observada entre atividade física e pressão arterial também fora observada por Stewart et al. (2005), que identificaram mudanças em indicadores de síndrome metabólica entre adultos após seis meses de treinamento, dentre as alterações, houve diminuição da gordura corporal total e da gordura abdominal, além disso, alterações na PAD ocorreram apenas no grupo que participou do programa proposto.

No que se refere à relação da atividade física com o indicador glicêmico utilizado, no estudo conduzido por Herbst et al. (2006) foi constatada a existência de uma correlação negativa entre a prática de exercício durante os dias da semana e a glicemia mensurada pelo método de hemoglobina glicosilada em pacientes pediátricos com diabetes tipo 1. De acordo com os autores, foi verificado que, dentre o índice de massa corpórea, freqüência de hipoglicemia, dosagem de insulina e freqüência de atividade física, esse último é o fator mais influente na glicemia dos pacientes. O FinnDiane Study observou correlação entre glicemia e prática de atividade física em mulheres, porém o mesmo resultado, diferentemente do observado em nosso estudo, 
não foi encontrado em homens (WADE'N et al., 2005).

Quando os fatores de risco foram analisados de maneira agregada, foi possível identificar que apenas as atividades realizadas no trabalho foram relacionadas negativamente com o escore de risco construído. Uma possível justificativa para tal achado diz respeito ao fato da amostra ter sido composta por educadores físicos, profissão essa que por natureza tem forte relação com a prática de atividades físicas. Mas ao analisar de maneira mais profunda os resultados, observa-se que a maior contribuição deste estudo reside no fato de identificar claramente que a atividade física é uma variável complexa e, como tal, deve ser analisada de maneira cuidadosa em suas diferentes facetas, neste caso específico, seus diferentes domínios.

Apesar dos resultados mostrarem correlações negativas significantes da prática de atividades físicas com a glicemia, PAD, PAS e \%GC, 0 instrumento utilizado para a mensuração das atividades habituais se resume a uma avaliação momentânea, enquanto o VO2máx, é um atributo que o indivíduo possui. Assim como no estudo de Herbst et al. (2006), a metodologia para verificar a prática de atividade física pode limitar a força dos resultados encontrados e desta maneira poderia ser indicada como uma das possíveis justificativas para as baixas correlações encontradas em nosso estudo.

\section{Conclusão}

Com base nos resultados apresentados é possível concluir que em indivíduos ditos saudáveis as atividades relacionadas ao trabalho tiveram significância estatística negativa com $\% G C, P A S, P A D$. Porém, a atividade física Total, que é o somatório de todos os domínios investigados, também se relacionou de maneira negativa com a glicemia em jejum e PAD. Assim, é possível identificar a necessidade de se analisar a atividade física desmembrada em seus diferentes domínios quando se trata de explorar suas relações com indicadores de saúde.

\section{Referências}

ABU-OMAR, K.; RÜTTEN, A. Relation of leisure time, occupational, domestic, and commuting physical activity to health indicators in Europe. Preventive Medicine; v.47, n.3, p. 319-23, 2008. Disponível em:

http://dx.doi.org/doi:10.1016/j.ypmed.2008.03.012 . Acesso em 31/12/2008.

BAECKE, J.A.; BUREMA, J.; FRIJTERS, J.E. A short questionnaire for the measurement of habitual physical activity in epidemiological studies. American Journal of Clinical Nutrition v.36 n.36, p. 936-942, 1982. Disponível em: http://www.ajcn.org/cgi/reprint/36/5/936. Acesso em 18/05/2005.

CHENOWETH, D.; LEUTZINGER, J. The economic cost of physical inactivity and excess weight in American adults. International Journal of Physical Activity and Health; v.3 n.2, p .14863, 2006. Disponível em:

http://www.humankinetics.com/ipah/viewarticle.cf m?aid=5684. Acesso em 02/04/2007.

CHRISTOFARO, D.G.; CASONATTO, J.; FERNANDES, R.A.; CUCATO, G.G.; GONÇALVES, C.G.; OLIVEIRA, A.R.; POLITO, M.D. Efeito da duração do exercício aeróbio sobre as respostas hipotensivas agudas pósexercício. Revista da SOCERJ. v.21, n.6, p.404408, 2008. Disponível em:

http://sociedades.cardiol.br/soceri/revista/2008 0 6/a2008 v21 n06 a07JulianoCasonatto.pdf. Acesso em: 20/12/2008

CHRISTOFARO, D.G.; FERNANDES, R.A.; GERAGE, A.M.; ALVES, M.J.; POLITO, M.D.; OLIVEIRA, A.R. Validação do monitor de medida de pressão arterial Omron HEM 742 em adolescentes. Arquivos Brasileiros de Cardiologia v. 92, n.1, p. 10-15, 2009. Disponível em: http://dx.doi.org/doi:10.1590/S0066782X2009000100003. Acesso em: 06/07/2009.

EKELUND, U.; BRAGE, S.; FROBERG, K.; HARRO, M.; ANDERSSEN, S.A.; SARDINHA, L.B.; RIDDOCH, C.; ANDERSEN, L.B. TV viewing and physical activity are independently associated with metabolic risk in children: the European Youth Heart Study. Plos Medicine, v.3, n.12, p.2449-2457. 2006. Disponível em: http://dx.doi.org/doi:10.1371/journal.pmed.003048 8. Acesso em: 13/03/2007.

Executive summary of the third report of the National Cholesterol Education Program (NCEP) expert panel on detection, evaluation, and treatment of high blood cholesterol in adults (Adult Treatment Panel III). Journal of the American Medical Association 2001;285:248697.Disponível em: http://jama.amaassn.org/cgi/content/extract/285/19/2486. Acesso em 12/01/2005

FERNANDES, R.A.; OLIVEIRA, A.R.; FREITAS JÚNIOR, I.F. Correlação entre diferentes indicadores de adiposidade corporal e atividade física habitual em jovens do sexo masculino.

Revista Brasileira de Cineantropometria e Desempenho Humano V. 8, n.4 , p.32- 8, 2006. Disponível em: http://www.periodicos.ufsc.br/index.php/rbcdh/arti cle/view/3929/3334. Acesso em 11/09/2007.

GUTIN, B.; YIN, Z.; HUMPHRIES, M.C.;

BARBEAU, P. Relations of moderate and 
vigorous physical activity to fitness and fatness in adolescents. American Journal of Clinical

Nutritrion; V.81, n.4, p.746-50, 2005. Disponível em: http://www.ajcn.org/cgi/reprint/81/4/746

Acesso em 13/08/2008.

HALLAL, P.C.; DUMITH, S.C.; BASTOS, J.P.; REICHERT, F.F.; SIQUEIRA, F.V.; AZEVEDO, M.R. Evolution of the epidemiological research on physical activity in Brazil: a systematic review.

Revista de Saude Publica. v.41, n.3, p. 453-60, 2007. Disponível em:

http://dx.doi.org/doi:10.1590/S0034-

89102007000300018 . Acesso em 24/11/2008.

HERBST, A.; BACHRAN, R.; KAPELLEN, T.;

HOLL, R.W. Effects of regular physical activity on control of glycemia in pediatrics patients with type 1 diabetes mellitus. Archives of Pediatric Adolescence Medicine V.160, n. 6, p.573-77, 2006. Disponível em: http://archpedi.amaassn.org/cgi/reprint/160/6/573. Acesso em 26/06/2007.

HEYWARD, H.; STOLARCZYK, L.M. Avaliação da composição corporal aplicada. São Paulo Editora Manole 2000.

KANG, H.S.; GUTIN, B.; BARBEAU, P.; OWENS, S.; LEMMON, C.R.; ALLISON, J.; LITAKER, M.S.; LE, N.A. Physical training improves insulin resistance syndrome markers in obese adolescents. Medicine and Science in Sports \& Exercise; V.34, n. 12, p.1920 -7, 2002.

Disponível em: http://www.acsm$\mathrm{msse} .0 \mathrm{rg} / \mathrm{pt} / \mathrm{re} / \mathrm{msse} / \mathrm{abstract.00005768-}$ 200212000 00010.htm;jsessionid=JctbQH20zvxnT9L8cYBQZ pfk1GRVQM1bpJnK6QTHQ7TDVhCvRp5v!2118404334!181195629!8091!-1. Acesso em 30/01/2003.

LOHMAN, T.G.; ROCHE, A.F.; MARTOREL, R., editors. Anthropometric standardization reference manual. Champaign: Human Kinetics Books; 1988. p. 3-8.

ORAKZAI, R.H.; ORAKZAI, S.H.; NASIR, K.; ROGUIN, A.; PIMENTEL, I.; CARVALHO, J.A.; MENEGHELLO, R.; BLUMENTHAL, R.S.; SANTOS, R.D. Association of increased cardiorespiratory fitness with low risk for clustering of metabolic syndrome components in asymptomatic men. Archives of Medical Research. V.37, n.4, p. 522-8, 2006. Disponível em:

http://dx.doi.org/doi:10.1016/j.arcmed.2005.08.00 4 Acesso em 23/10/2007.

STEWART, K.J.; BACHER, A.C.; TURNER, K.L.; FLEG, J.L.; HEES, P.S.; SHAPIRO, E.P.; TAYBACK, M.; OUYANG, P. Effect of exercise on blood pressure in older persons: a randomized controlled trial. Archives of Internal Medicine. v.165, n. 7, p.756-62, 2005. Disponível em: http://archinte.ama-assn.org/cgi/reprint/165/7/756. Acesso em 19/02/2006.

SUN, S.S.; CHUMLEA, W.C.; HEYMSFIELD, S.B.; LUKASKI, H.C.; SCHOELLER, D.; FRIEDL, K.; KUCZMARSKI, R.J.; FLEGAL, K.M.; JOHNSON, C.L.; HUBBARD, V.S. Development of bioelectrical impedance analysis prediction equations for body composition with the use of a multicomponent model for use in epidemiologic surveys. American Journal of Clinical Nutrition. v.77, n.2, p. 331-40, 2003. Disponível em: http://www.ajcn.org/cgi/reprint/77/2/331. Acesso em 12/01/2004.

WADÉN, J.; TIKKANEN, H.; FORSBLOM, C.; FAGERUDD, J.; PETTERSSON-FERNHOLM, K.; LAKKA, T.; RISKA, M.; GROOP, P.H.;

FINNDIANE STUDY GROUP. Leisure time physical activity is associated with poor glycemic control in type 1 diabetic women: the FinnDiane study. Diabetes Care. V.28, n.4, p.777-82, 2005. Disponível em:

http://care.diabetesjournals.org/cgi/reprint/28/4/77 7. Acesso em 27/08/2006.

Esse artigo foi apresentado em Sessão Temática no VI Congresso Internacional de Educação Física e Motricidade Humana e XII Simpósio Paulista de Educação Física, realizado pelo Departamento de Educação Física do IB/UNESP Rio Claro, SP de 30/4 a 03/5 de 2009.

Endereço:

Ismael Forte Freitas Júnior

Rua Roberto Simonsen, 305.

Presidente Prudente SP Brasil

19060-900

Telefone: (18) 3229-5345 Fax: (18) 3221-5681

e-mail: ismael@fct.unesp.br

Recebido em: 10 de fevereiro de 2009.

Aceito em: 03 de abril de 2009.

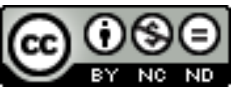

Motriz. Revista de Educação Física. UNESP, Rio Claro, SP, Brasil - elSSN: 1980-6574 - está licenciada sob Licenca Creative Commons 